\title{
LA DEUXIÈME CONJECTURE DE HIRSCHFELD-ŻELAZKO POUR LES ALGĖBRES DE BANACH EST FAUSSE
}

\author{
BERNARD AUPETIT ${ }^{1}$
}

\begin{abstract}
We prove that there exists a noncommutative complex Banach algebra with no quasi-nilpotents elements such that the spectrum function is continuous for the Hausdorff metric.
\end{abstract}

Introduction. Dans [7], R. A. Hirschfeld et W. Żelazko posèrent le problème suivant: est-ce qu'une algèbre de Banach complexe, avec unité, où le rayon spectral $\rho$ est continu et sans éléments quasi-nilpotents-c'est-à-dire que $\rho(x)$ $=0$ implique $x=0$-est commutative? Par la suite, diverses personnes ([4], [5], [6]) donnèrent des exemples d'algèbres non commutatives sans éléments quasi-nilpotents, mais sans rien spécifier sur le spectre. L'objet de cette note est de montrer que cette conjecture est fausse. Ainsi la seule façon simple de caractériser les algèbres de Banach commutative est de supposer $\rho$ uniformément continu, voir [2].

THÉORÈME. Il existe une algèbre de Banach complexe avec unité $A$, non commutative, sans éléments quasi-nilpotents, telle que $x \mapsto \mathrm{Sp} x$ soit continue sur A.

Corollaire. Il existe une algèbre de Banach complexe avec unité, non commutative, sans éléments quasi-nilpotents, vérifiant une identité polynômiale.

Démonstration du Théorème. Soit $U$ le disque unité ouvert de $\mathbf{C}, \boldsymbol{B}$ l'algèbre de Banach commutative des fonctions sur $\bar{U} \times \bar{U}$, holomorphes sur $U \times U$. Dans l'algèbre de Banach $M_{2}(B)$, pour la norme définie par:

$$
\left\|\left(\begin{array}{l}
a, b \\
c, d
\end{array}\right)\right\|=\operatorname{Max}(\|a\|+\|b\|,\|c\|+\|d\|)
$$

où $a, b, c, d \in B$ et où \|\| désigne la norme uniforme sur $B$, soit $A$ la sous-algèbre fermée, non commutative, avec unité, formée par les matrices

$$
\left(\begin{array}{cc}
f\left(z_{1}, z_{2}\right), & g\left(z_{1}, z_{2}\right) \\
\left(z_{1}+z_{2}\right) \operatorname{Tg}\left(z_{1}, z_{2}\right), & \operatorname{Tf}\left(z_{1}, z_{2}\right)
\end{array}\right)
$$

Received by the editors April 20, 1976.

AMS (MOS) subject classifications (1970). Primary 46H05, 46H20; Secondary $46 \mathrm{~J} 35$.

Key words and phrases. Commutative Banach algebra, quasi-nilpotent elements, spectrum, spectral radius, continuity of the spectrum, irreducible representations.

${ }^{1}$ Ce travail a été subventionné par le Conseil national de recherches du Canada (A 7668). 
où $f, g \in B$ et où $T$ est l'automorphisme isométrique de $B$ défini par $T f\left(z_{1}, z_{2}\right)=f\left(z_{2}, z_{1}\right)$. D'après [1], $x \mapsto \operatorname{Sp} x$ est continue sur $A$. Si $m \in A$ est quasi-nilpotent alors, toujours d'après [1], $\{0\}=\cup_{x \in X(B)} \operatorname{Sp}(\hat{\chi}(m))$, où

$$
\hat{\chi}(m)=\left[\begin{array}{cc}
\chi\left(f\left(z_{1}, z_{2}\right)\right), & \chi\left(g\left(z_{1}, z_{2}\right)\right) \\
\chi\left(\left(z_{1}+z_{2}\right) \operatorname{Tg}\left(z_{1}, z_{2}\right)\right), & \chi\left(T f\left(z_{1}, z_{2}\right)\right)
\end{array}\right) \in M_{2}(\mathbf{C})
$$

et où $X(B)$ est l'ensemble des caractères de $B$. Aussi $\hat{\chi}(m)$ est quasi-nilpotente, donc de carré nul. Comme $B$ est sans radical, $m^{2}=0$, donc on a $f\left(z_{1}, z_{2}\right)^{2}+\left(z_{1}+z_{2}\right) g\left(z_{1}, z_{2}\right) g\left(z_{2}, z_{1}\right)=0$, ce qui implique que l'on a $f\left(z_{1}, z_{2}\right) \equiv g\left(z_{1}, z_{2}\right) \equiv 0$, aussi $m=0$.

RemarQue. Si nous avions pris l'identité au lieu de $T$, la fin de la démonstration aurait été plus facile, mais l'algèbre aurait été commutative. C'est pourquoi l'utilisation de $T$ et des fonctions de deux variables a pour but de rendre $A$ non commutative.

Démonstration du Corollaire. Si $a, b, c \in M_{2}(B)$, alors, d'après le théorème de Hamilton-Cayley, on a $\left[\hat{\chi}(a),[\hat{\chi}(b), \hat{\chi}(c)]^{2}\right]=0$, pour tout $\chi$, où $\left[\right.$, ] désigne le commutateur, donc $\left[a,[b, c]^{2}\right]=0$, et en particulier c'est vrai sur $A$. $\square$ Pour plus de détails, voir [3].

\section{BIBLIOGRAPHY}

1. S. T. M. Ackermans, $A$ case of strong spectral continuity, Nederl. Akad. Wetensch. Proc. A71 = Indag. Math. 30 (1968), 455-459. MR 40 \#39.

2. B. Aupetit, Caractérisation spectrale des algèbres de Banach commutatives, Pacific J. Math. 63 (1976), 23-35. MR 54 \# 3409.

3. _ Propriétés spectrales des algèbres de Banach (to appear).

4. H. Behncke, Nilpotent elements in group algebras, Bull. Acad. Polon. Sci. 19 (1971), 197-198. MR 44 \#813.

5. J. Duncan and A. W. Tullo, Finite dimensionality, nilpotents and quasinilpotents in Banach algebras, Proc. Edinburgh Math. Soc. (2) 19 (1974/75), 45-49. MR 49 \#9631.

6. R. A. Hirschfeld and S. Rolewicz, A class of non-commutative Banach algebras without divisors of zero, Bull. Acad. Polon. Sci. 17 (1969), 751-753. MR 40 \#802.

7. R. A. Hirschfeld and W. Zelazko, On spectral norm Banach algebras, Bull. Acad. Polon. Sci. 16 (1968), 195-199. MR 37 \#4621.

Département de Mathématiques, Université Laval, QuÉbec, Canada 